\title{
A note on the stability of pencils of plane curves
}

\author{
Aline Zanardini ${ }^{1}$ \\ Received: 3 March 2021 / Accepted: 18 July 2021 / Published online: 30 August 2021 \\ (c) The Author(s) 2021
}

\begin{abstract}
We investigate the problem of classifying pencils of plane curves of degree $d$ up to projective equivalence. We obtain explicit stability criteria in terms of the log canonical threshold by relating the stability of a pencil to the stability of the curves lying on it.
\end{abstract}

Keywords Pencils of plane curves · GIT stability · Log canonical threshold

Mathematics Subject Classification Primary 14L24; Secondary 14E99

\section{Introduction}

The group $P G L(3)$ acts naturally on the space of all pencils of curves of degree $d$ in $\mathbb{P}^{2}$ and in order to construct the corresponding classification space (via geometric invariant theory), a fundamental problem consists in explicitly describing what the (semi)stable pencils are, with respect to the action. In this paper we obtain explicit stability criteria in terms of some known invariants of singularities. We relate the stability of a pencil $\mathcal{P}$ to the number

$$
l_{c t}\left(\mathbb{P}^{2}, \mathcal{C}_{d}\right)=\sup \left\{c \in \mathbb{Q} ;\left(\mathbb{P}^{2}, c \mathcal{C}_{d}\right) \text { is log canonical at } p\right\}
$$

which is known as the log canonical threshold of the pair $\left(\mathbb{P}^{2}, \mathcal{C}_{d}\right)$ at $p$, where $\mathcal{C}_{d}$ is a curve in $\mathcal{P}$ and $p$ is a base point (see [7, Section 8]). We also relate the stability of $\mathcal{P}$ to the global $\log$ canonical threshold of the pair $\left(\mathbb{P}^{2}, \mathcal{C}_{d}\right)$, i.e. the number $l c t\left(\mathbb{P}^{2}, \mathcal{C}_{d}\right)=\min _{p \in \mathcal{C}_{d}} l c t_{p}\left(\mathbb{P}^{2}, \mathcal{C}_{d}\right)$ (cf. Definition 2.4); and to the multiplicities of its generators at a base point.

Letting $\mathscr{P}_{d}$ denote the space of all pencils of plane curves of degree $d$, our main results are given by Theorems 1.1, 1.2 and 1.3 below.

Theorem 1.1 (= Theorem 4.7) Let $\mathcal{P}$ be a pencil in $\mathscr{P}_{d}$ containing a curve $C_{f}$ such that lct $\left(\mathbb{P}^{2}, C_{f}\right)=\alpha$. If $\mathcal{P}$ is unstable (resp. not stable), then $\mathcal{P}$ contains a curve $C_{g}$ such that $\operatorname{lct}\left(\mathbb{P}^{2}, C_{g}\right)<\frac{3 \alpha}{2 d \alpha-3}($ resp. $\leq)$.

Theorem 1.2 (= Theorem 4.10) If $\mathcal{P} \in \mathscr{P}_{d}$ is semi-stable (resp. stable), then lct $p\left(\mathbb{P}^{2}, C_{f}\right) \geq$ $\frac{3}{2 d}$ (resp. $>$ ) for any curve $C_{f}$ in $\mathcal{P}$ and any base point $p$.

Aline Zanardini

a.zanardini@math.leidenuniv.nl

1 Mathematical Institute, Leiden University, Leiden, The Netherlands 
Theorem 1.3 (= Corollary 3.6) Let $\mathcal{P}$ be a pencil in $\mathscr{P}_{d}$. If we can find two curves $C_{f}$ and $C_{g}$ in $\mathcal{P}$ such that mult ${ }_{p}\left(C_{f}\right)+$ mult $_{p}\left(C_{g}\right)>\frac{4 d}{3}$ (resp. $\geq$ ) for some base point $p$, then $\mathcal{P}$ is unstable (resp. not stable).

In particular, we extend and idea of Hacking [4] and Kim-Lee [5] who observed the following connection between two notions of stability, one coming from geometric invariant theory and the other coming from the Minimal Model Program: if $H \subset \mathbb{P}^{n}$ is a hypersurface of degree $d$ and the pair $\left(\mathbb{P}^{n}, \frac{n+1}{d} H\right)$ is log canonical, then $H$ is semi-stable for the natural action of $P G L(n+1)$. And if $\left(\mathbb{P}^{n},\left(\frac{n+1}{d}+\varepsilon\right) H\right)$ is log canonical for some $0<\varepsilon \ll 1$, then $H$ is stable. Moreover, for $d=3$ and $d=4$, we recover some of the results from [9] and [1], respectively.

One of the key ingredients in our approach consists in observing that we can sometimes determine whether a pencil $\mathcal{P} \in \mathscr{P}_{d}$ is (semi)stable or not by looking at the stability of its generators. We also prove Theorems $1.4,1.5$ and 1.6 below:

Theorem 1.4 (= Corollary 3.12) If a pencil $\mathcal{P} \in \mathscr{P}_{d}$ has only semi-stable (resp. stable) members, then $\mathcal{P}$ is semi-stable (resp. stable).

Theorem 1.5 (= Theorem 3.14) If $\mathcal{P} \in \mathscr{P}_{d}$ contains at most one strictly semi-stable curve (and all other curves in $\mathcal{P}$ are stable), then $\mathcal{P}$ is stable.

Theorem 1.6 (= Theorem 3.15) If $\mathcal{P} \in \mathscr{P}_{d}$ contains at most two semi-stable curves $C_{f}$ and $C_{g}$ (and all other curves in $\mathcal{P}$ are stable), then $\mathcal{P}$ is strictly semi-stable if and only if there exists a one-parameter subgroup $\lambda$ such that $C_{f}$ and $C_{g}$ are both non-stable with respect to this $\lambda$.

The paper is organized as follows: Sect. 2 contains the relevant background material on $\log$ canonical pairs and on geometric invariant theory. In Sect. 3 we describe the stability criterion of Hilbert-Mumford for pencils of plane curves and we relate the stability of a pencil to the stability of its generators. Then, in Sect. 4 we use the notations and results from Sect. 3 to relate the stability of a pencil to the log canonical threshold.

We work over $\mathbb{C}$ throughout.

\section{Background}

For the convenience of the reader, we begin presenting some basic notions concerning log canonical pairs and the relevant background on geometric invariant theory.

\subsection{The log canonical threshold}

We first introduce the log canonical threshold, which will play an important role in our analysis of the stability of pencils of plane curves (Sect. 4). We refer to [7, Section 8] for a more detailed exposition.

Let $X$ be a normal algebraic variety of dimension $n$ and let $\Delta=\sum d_{i} D_{i}$ be an effective $\mathbb{Q}$-divisor in $X$, i.e. a $\mathbb{Q}$-linear combination of prime divisors with non-negative coefficients.

Definition 2.1 Given any birational morphism $\mu: \tilde{X} \rightarrow X$, with $\tilde{X}$ normal, we write $K_{\tilde{X}} \equiv$ $\mu^{*}\left(K_{X}+\Delta\right)+\sum a_{E} E$, where $E \subset \tilde{X}$ are distinct prime divisors, $a_{E} \doteq a(E, X, \Delta)$ are the discrepancies of $E$ with respect to $(X, \Delta)$ and a non-exceptional divisor $E$ appears in the sum if and only if $E=\mu_{*}^{-1} D_{i}$ for some $i$ (in that case with coefficient $a(E, X, \Delta)=-d_{i}$ ). 
Definition 2.2 A log resolution of the pair $(X, \Delta)$ consists of a proper birational morphism $\mu: \tilde{X} \rightarrow X$ such that $\tilde{X}$ is smooth and $\mu_{*}^{-1}(\Delta) \cup \operatorname{Exc}(\mu)$ is a simple normal crossings divisor (that is, each component is smooth and each point étale locally looks like the intersection of $r \leq n$ coordinate hyperplanes).

Definition 2.3 We say $(X, \Delta)$ is $\log$ canonical (lc) if $K_{X}+\Delta$ is $\mathbb{Q}$-Cartier and given any $\log$ resolution $\mu: \tilde{X} \rightarrow X$ we have $K_{\tilde{X}} \equiv \mu^{*}\left(K_{X}+\Delta\right)+\sum a_{E} E$ with all the discrepancies satisfying $a_{E} \geq-1$. In particular, if $X$ is smooth and $\Delta=d_{i} D_{i}$ is simple normal crossings, then $(X, \Delta)$ is $\log$ canonical if and only if $d_{i} \leq 1$ for all $i$.

Definition 2.4 The number $l \operatorname{ct}(X, \Delta) \doteq \sup \{t ;(X, t \Delta)$ is $\log$ canonical $\}$ is called the $\log$ canonical threshold of $(X, \Delta)$.

Remark 2.5 We can also consider a local version, $\operatorname{lct}_{p}(X, \Delta)$, taking the supremum over all $t$ such that $(X, t \Delta)$ is log canonical in an open neighborhood of $p$, where $p \in X$ is a closed point.

\subsection{Geometric invariant theory}

We now recall the relevant definitions and basic results from geometric invariant theory, and we point the reader to [3] for more details. The general setup consists of a reductive group $G$ acting on an algebraic variety $X$, and we first consider the simple case when $X \simeq \mathbb{C}^{n+1}$.

Definition 2.6 A point $x \in X$ is said to be semi-stable for the $G$-action if and only if $0 \notin \overline{G \cdot x}$.

Definition 2.7 A point $x \in X$ is said to be stable for the $G$-action if and only if the following two conditions hold:

(i) The orbit $G \cdot x \subset X$ is closed and

(ii) The stabilizer $G_{x} \leq G$ is finite

When $X \hookrightarrow \mathbb{P}^{n}$ is a projective variety, a point $x \in X$ will be called semi-stable (resp. stable) if any point $\tilde{X} \in \mathbb{C}^{n+1}$ lying over $x$ is semi-stable (resp. stable). Moreover, a point $x \in X$ will be called unstable if $x$ is not semi-stable.

From now on we assume that $X$ is a projective variety, embedded in some projective space.

Given a one-parameter subgroup $\lambda: \mathbb{C}^{\times} \rightarrow G$ we may regard $\mathbb{C}^{n+1}$ as a representation of $\mathbb{C}^{\times}$. Since any representation of $\mathbb{C}^{\times}$is completely reducible and every irreducible representation is one dimensional, we can choose a basis $e_{0}, \ldots, e_{n}$ of $\mathbb{C}^{n+1}$ so that $\lambda(t) \cdot e_{i}=t^{r_{i}} e_{i}$, for some $r_{i} \in \mathbb{Z}$. Then, given $x \in X \hookrightarrow \mathbb{P}^{n}$ we can pick $\tilde{x} \in \operatorname{Cone}(X) \subset \mathbb{C}^{n+1}$ lying above $x$ and write $\tilde{x}=\sum x_{i} e_{i}$ with respect to this basis so that $\lambda(t) \cdot x \doteq \lambda(t) \cdot \tilde{x}=\sum t^{r_{i}} x_{i} e_{i}$. The weights of $x$ are the set of integers $r_{i}$ for which $x_{i}$ is not zero.

These notations allow us to define the so called Hilbert-Mumford weight of a point $x \in X$ :

Definition 2.8 Given $x \in X$ and a one-parameter subgroup $\lambda: \mathbb{C}^{\times} \rightarrow G$, we define the Hilbert-Mumford weight of $x$ at $\lambda$ to be $\mu(x, \lambda) \doteq \min \left\{r_{i}: x_{i} \neq 0\right\}$.

Remark 2.9 The Hilbert-Mumford weight satisfies the following properties:

(i) $\mu\left(x, \lambda^{n}\right)=n \mu(x, \lambda)$ for all $n \in \mathbb{N}$

(ii) $\mu\left(g \cdot x, g \lambda g^{-1}\right)=\mu(x, \lambda)$ for all $g \in G$ 
The known numerical criterion for stability can thus be stated:

Theorem 2.10 (Hilbert-Mumford criterion) Let $G$ be a reductive group acting linearly on a projective variety $X \hookrightarrow \mathbb{P}^{n}$. Then for a point $x \in X$ we have that $x$ is semi-stable (resp. stable) if and only if $\mu(x, \lambda) \leq 0$ (resp. $<$ ) for all one-parameter subgroups $\lambda$ of $G$.

That is, a point $x \in X$ is unstable (resp. not stable) for the $G$-action if and only if there exists a one-parameter subgroup $\lambda: \mathbb{C}^{\times} \rightarrow G$ for which all the weights of $x$ are all positive (resp. non-negative).

In this paper we are interested in the case where $G$ is the group $P G L(3)$ and $X$ is the space $\mathscr{P}_{d}$ of pencils of plane curves of degree $d$, embedded via Plücker coordinates in projective space.

\section{Stability criterion for pencils of plane curves}

As in [9], we view a pencil of plane curves of degree $d$ as a choice of line in the space of all plane curves of degree $d$. We identify the space $\mathscr{P}_{d}$ of all such pencils with the Grassmannian $G r\left(2, S^{d} V^{*}\right)$, where $V \doteq H^{0}\left(\mathbb{P}^{2}, \mathcal{O}_{\mathbb{P}^{2}}(1)\right)$, which we further embed in $\mathbb{P}\left(\Lambda^{2} S^{d} V^{*}\right)$ via the Plücker embedding. The group $P G L(V)$ acts naturally on $V$, hence on the invariant subvariety $\mathscr{P}_{d}$, and our goal in this Section is to describe the corresponding (semi)stable points for the action.

It turns out that we are able to partially determine whether a pencil $\mathcal{P} \in \mathscr{P}_{d}$ is (semi)stable or not by looking at the stability of its generators. Therefore, from now on we will consider the actions of $P G L(V)$ on both $\mathscr{P}_{d}$ and $S^{d} V^{*}$, the space of plane curves of degree $d$.

The numerical criterion of Hilbert-Mumford (Theorem 2.10) tells us we need to know how the diagonal elements of $P G L(V)$ act, and since both $P G L(V)$ and $S L(V)$ act with the same orbits, we will focus on the action of diagonal elements of the latter.

Note that if we choose a pencil $\mathcal{P} \in \mathscr{P}_{d}$ and two curves $C_{f}$ and $C_{g}$ as generators, these represented (in some choice of coordinates) by $f=\sum f_{i j} x^{i} y^{j} z^{d-i-j}=0$ and $g=\sum g_{i j} x^{i} y^{j} z^{d-i-j}=0$, respectively; then the Plücker coordinates of $\mathcal{P}$ are given by all the $2 \times 2$ minors $m_{i j k l} \doteq\left|\begin{array}{ll}f_{i j} & f_{k l} \\ g_{i j} & g_{k l}\end{array}\right|$. Thus, the action of $\left(\begin{array}{ccc}\alpha & 0 & 0 \\ 0 & \beta & 0 \\ 0 & 0 & \gamma\end{array}\right) \in S L(V)$ on the Plücker coordinates is given by

$$
\left(m_{i j k l}\right) \mapsto\left(\alpha^{i+k} \beta^{j+l} \gamma^{2 d-i-j-k-l} m_{i j k l}\right)
$$

In order to obtain the desired stability criteria from Theorems 1.1 and 1.2, the first step in our approach consists in introducing an "affine" analogue of the Hilbert-Mumford weight (see Definition 2.8) and then translating the Hilbert-Mumford criterion in terms of this quantity (Proposition 3.2). The definition is as follows:

Definition 3.1 Given $\mathcal{P} \in \mathscr{P}_{d}$ and a one-parameter subgroup $\lambda$ of $S L(V)$, we define the affine weight of $\mathcal{P}$ at $\lambda$ to be

$$
\omega(\mathcal{P}, \lambda) \doteq \min \left\{\left(a_{x}-a_{z}\right)(i+k)+\left(a_{y}-a_{z}\right)(j+l): m_{i j k l} \neq 0\right\}
$$

where we choose coordinates in $\mathbb{P}^{2}$ so that $\lambda: \mathbb{C}^{\times} \rightarrow S L(V)$ is given by

$$
t \mapsto\left(\begin{array}{ccc}
t^{a_{x}} & 0 & 0 \\
0 & t^{a_{y}} & 0 \\
0 & 0 & t^{a_{z}}
\end{array}\right)
$$


for some weights $a_{x}, a_{y}, a_{z} \in \mathbb{Z}$ with $a_{x} \geq a_{y} \geq a_{z}, a_{x}>0$ and $a_{x}+a_{y}+a_{z}=0$

Stated in terms of $\omega(\mathcal{P}, \lambda)$, the Hilbert-Mumford criterion becomes:

Proposition 3.2 A pencil $\mathcal{P} \in \mathscr{P}_{d}$ is unstable (resp. not stable) if and only if there exists a one-parameter subgroup $\lambda: \mathbb{C}^{\times} \rightarrow S L(V)$ and a choice of coordinates in $\mathbb{P}^{2}$ such that $\omega(\mathcal{P}, \lambda)>\frac{2 d}{3}\left(a_{x}+a_{y}-2 a_{z}\right)($ resp. $\geq)$

Proof A pencil $\mathcal{P} \in \mathscr{P}_{d}$ is unstable (resp. not stable) if and only if there exists a oneparameter subgroup $\lambda: \mathbb{C}^{\times} \rightarrow S L(V)$ and a choice of coordinates in $\mathbb{P}^{2}$ satisfying that for any $i, j, k$ and $l$ such that $m_{i j k l} \neq 0$ (in those coordinates) we have $a_{x}(i+k)+a_{y}(j+l)+$ $a_{z}(2 d-i-j-k-l)>0($ resp. $\geq)$ if and only if

$$
\left(a_{x}-a_{z}\right)(i+k)+\left(a_{y}-a_{z}\right)(j+l)-\frac{2 d}{3}\left(a_{x}+a_{y}-2 a_{z}\right)>0 \quad(\text { resp. } \geq 0)
$$

Similarly, we define an affine weight for plane curves of degree $d$ :

Definition 3.3 Given a plane curve of degree $d C_{f}$ and a one-parameter subgroup $\lambda: \mathbb{C}^{\times} \rightarrow$ $S L(V)$ we define the affine weight of $f$ at $\lambda$ to be

$$
\omega(f, \lambda) \doteq \min \left\{\left(a_{x}-a_{z}\right) i+\left(a_{y}-a_{z}\right) j: f_{i j} \neq 0\right\}
$$

And for curves the Hilbert-Mumford criterion becomes:

Proposition 3.4 A curve $C_{f}$ is unstable (resp. not stable) if and only if there exists a oneparameter subgroup $\lambda: \mathbb{C}^{\times} \rightarrow S L(V)$ and a choice of coordinates in $\mathbb{P}^{2}$ such that $\omega(f, \lambda)>$ $\frac{d}{3}\left(a_{x}+a_{y}-2 a_{z}\right)($ resp. $\geq)$.

The inspiration for Definition 3.1 comes from [6, Definition 2.2] and it is justified by Corollary 4.2. Given a pencil $\mathcal{P} \in \mathscr{P}_{d}$ and a curve $C_{f} \in \mathcal{P}$, the idea will be to use this affine weight to bound the $\log$ canonical threshold of the pair $\left(\mathbb{P}^{2}, C_{f}\right)$ at a base point of $\mathcal{P}$, as well as the global log canonical threshold.

\subsection{The stability of the generators}

Given a pencil $\mathcal{P} \in \mathscr{P}_{d}$ and a curve $C_{f} \in \mathcal{P}$, it is interesting to compare the affine weights $\omega(f, \lambda)$ and $\omega(\mathcal{P}, \lambda)$ for a fixed one-parameter subgroup $\lambda$. We state and prove a series of results in this direction that allow us to relate the stability of a pencil to the stability of its generators (Corollary 3.12 and Theorems 3.14 and 3.15).

Even when omitted, we will always choose coordinates $[x, y, z]$ in $\mathbb{P}^{2}$ so that a oneparameter subgroup $\lambda: \mathbb{C}^{\times} \rightarrow S L(V)$ is normalized as in (1).

Proposition 3.5 Given a pencil $\mathcal{P} \in \mathscr{P}_{d}$ and any two (distinct) curves $C_{f}, C_{g} \in \mathcal{P}$ we have that $\omega(f, \lambda) \leq \omega(f, \lambda)+\omega(g, \lambda) \leq \omega(\mathcal{P}, \lambda)$, for all one-parameter subgroups $\lambda: \mathbb{C}^{\times} \rightarrow$ $S L(V)$.

Proof Given $\mathcal{P}$ and $\lambda: \mathbb{C}^{\times} \rightarrow S L(V)$, choose coordinates in $\mathbb{P}^{2}$ that normalize $\lambda$ and choose any two curves $C_{f}$ and $C_{g}$ of $\mathcal{P}$ so that $\mathcal{P}$ is represented by the Plücker coordinates $m_{i j k l}=f_{i j} g_{k l}-g_{i j} f_{k l}$. 
Let $i, j, k$ and $l$ be such that $m_{i j k l}=f_{i j} g_{k l}-g_{i j} f_{k l} \neq 0$ and

$$
\omega(\mathcal{P}, \lambda)=\left(a_{x}-a_{z}\right)(i+k)+\left(a_{y}-a_{z}\right)(j+l)
$$

Then either $i$ and $j$ are such that $f_{i j} \neq 0$ or $k$ and $l$ are such that $f_{k l} \neq 0$. In the first case there are two possibilities: either $g_{k l}=0$, which implies $g_{i j} \neq 0$ and $f_{k l} \neq 0$; or $g_{k l} \neq 0$. Similarly, in the second case either $g_{i j}=0$, which implies $g_{k l} \neq 0$ and $f_{i j} \neq 0$; or $g_{i j} \neq 0$.

In any case we have

$$
\begin{aligned}
\left(a_{x}-a_{z}\right)(i+k)+\left(a_{y}-a_{z}\right)(j+l)= & \left(\left(a_{x}-a_{z}\right) i+\left(a_{y}-a_{z}\right) j\right)+ \\
& +\left(\left(a_{x}-a_{z}\right) k+\left(a_{y}-a_{z}\right) l\right) \\
\geq & \omega(f, \lambda)+\omega(g, \lambda)
\end{aligned}
$$

As a consequence, we can relate the stability of a pencil $\mathcal{P} \in \mathscr{P}_{d}$ to the multiplicity of its generators at a base point.

Corollary 3.6 Let $\mathcal{P}$ be a pencil in $\mathscr{P}_{d}$ with generators $C_{f}$ and $C_{g}$. If there exists a base point $p$ of $\mathcal{P}$ such that mult $p\left(C_{f}\right)+$ mult $_{p}\left(C_{g}\right)>\frac{4 d}{3}$ (resp. $\geq$ ), then $\mathcal{P}$ is unstable (resp. not stable).

Proof If $P$ is any base point of $\mathcal{P}$, we can always choose coordinates so that we have $p=(0$ : $0: 1)$. Letting $a_{x}=1, a_{y}=1, a_{z}=-2$ and $\lambda$ be the corresponding one-parameter subgroup (which in these coordinates is normalized as in (1)), we have that $\omega(f, \lambda)=3 \cdot \operatorname{mult}_{p}\left(C_{f}\right)$ and $\omega(g, \lambda)=3 \cdot$ mult $_{p}\left(C_{g}\right)$ for any choice of generators of $\mathcal{P}$, say $C_{f}$ and $C_{g}$. These two equalities, together with Proposition 3.5, imply

$$
\frac{\omega(\mathcal{P}, \lambda)}{\left(a_{x}-a_{z}\right)+\left(a_{y}-a_{z}\right)} \geq 3 \cdot \frac{\operatorname{mult}_{p}\left(C_{f}\right)+\operatorname{mult}_{p}\left(C_{g}\right)}{\left(a_{x}-a_{z}\right)+\left(a_{y}-a_{z}\right)}
$$

and since $\left(a_{x}-a_{z}\right)+\left(a_{y}-a_{z}\right)=6$, the result follows from the Hilbert-Mumford criterion (Proposition 3.2).

Remark 3.7 In [2, Lemma 3.3] it is proved that a plane curve of degree $d$, say $\mathcal{C}_{d}$, satisfying mult $\left.\mathcal{C}_{d}\right)>\frac{2 d}{3}$ is unstable. Our proof of Corollary 3.6 above is an adaptation of the argument therein.

Example 3.8 Assume $d<6$. Let $\mathcal{P} \in \mathscr{P}_{d}$ be a pencil which contains a curve $\mathcal{C}_{d}$ consisting of $d$ lines through a common point $p$, and which contains another curve with a double point at $p$. Then $\mathcal{P}$ is unstable.

Note that the content of Proposition 3.5 gives a lower bound for the affine weight $\omega(\mathcal{P}, \lambda)$. We can also find an upper bound:

Proposition 3.9 Given $\mathcal{P} \in \mathscr{P}_{d}$, a one-parameter subgroup $\lambda: \mathbb{C}^{\times} \rightarrow S L(V)$ and any curve $C_{f} \in \mathcal{P}$ there exists a curve $C_{g}$ in $\mathcal{P}$ such that

$$
\omega(\mathcal{P}, \lambda) \leq \omega(f, \lambda)+\omega(g, \lambda)
$$

Proof Fix a one-parameter subgroup $\lambda: \mathbb{C}^{\times} \rightarrow S L(V)$ and coordinates in $\mathbb{P}^{2}$ that normalize $\lambda$. Choose any two curves $C_{f}$ and $C_{g}$ of $\mathcal{P}$. Let $i$ and $j$ be such that $f_{i j} \neq 0$ and $\omega(f, \lambda)=$ $\left(a_{x}-a_{z}\right) i+\left(a_{y}-a_{z}\right) j$. Replacing $g$ by $g^{\prime}=g-\frac{g_{i j}}{f_{i j}} f$ we have $g_{i j}=0$, hence $m_{i j k l} \neq 0$ for all $k$ and $l$ such that $g_{k l} \neq 0$ and it follows that $\omega(\mathcal{P}, \lambda) \leq \omega(f, \lambda)+\omega(g, \lambda)$. 
Corollary 3.10 Given $\mathcal{P} \in \mathscr{P}_{d}$, a one-parameter subgroup $\lambda: \mathbb{C}^{\times} \rightarrow S L(V)$ and any curve $C_{f} \in \mathcal{P}$ there exists a curve $C_{g}$ in $\mathcal{P}$ such that

$$
\omega(\mathcal{P}, \lambda) \leq 2 \max \{\omega(f, \lambda), \omega(g, \lambda)\}
$$

Corollary 3.11 Given $\mathcal{P} \in \mathscr{P}_{d}$, a one-parameter subgroup $\lambda: \mathbb{C}^{\times} \rightarrow S L(V)$ and any curve $C_{f} \in \mathcal{P}$ there exists a curve $C_{g}$ in $\mathcal{P}$ such that

$$
\omega(\mathcal{P}, \lambda)=\omega(f, \lambda)+\omega(g, \lambda)
$$

Corollary 3.12 If a pencil $\mathcal{P} \in \mathscr{P}_{d}$ has only semi-stable (resp. stable) members, then $\mathcal{P}$ is semi-stable (resp. stable).

Corollary 3.13 If a pencil $\mathcal{P} \in \mathscr{P}_{d}$ contains only plane curves $C_{d}$ such that the pairs $\left(\mathbb{P}^{2}, 3 / d C_{d}\right)$ (resp. $\left.\left(\mathbb{P}^{2},(3 / d+\varepsilon) C_{d}\right), 0<\varepsilon<<1\right)$ are log canonical, then $\mathcal{P}$ is semistable (resp. stable).

Proof As observed in [4] and [5], in this case all members of $\mathcal{P}$ are semi-stable (resp. stable).

As a result of our comparison between $\omega(f, \lambda)$ and $\omega(\mathcal{P}, \lambda)$ we can now prove Theorems 3.14 and 3.15 below:

Theorem 3.14 If $\mathcal{P} \in \mathscr{P}_{d}$ contains at most one strictly semi-stable curve (and all other curves in $\mathcal{P}$ are stable), then $\mathcal{P}$ is stable.

Proof Given $\mathcal{P}$ as above, if all curves in $\mathcal{P}$ are stable, then $\mathcal{P}$ is stable by Corollary 3.12. Otherwise, let $C_{f}$ be the unique strictly semi-stable curve in $\mathcal{P}$. Given any one-parameter subgroup $\lambda$, by Proposition 3.9 there exists a curve $C_{g}$ such that

$$
\frac{\omega(\mathcal{P}, \lambda)}{\left(a_{x}-a_{z}\right)+\left(a_{y}-a_{z}\right)} \leq \frac{\omega(f, \lambda)}{\left(a_{x}-a_{z}\right)+\left(a_{y}-a_{z}\right)}+\frac{\omega(g, \lambda)}{\left(a_{x}-a_{z}\right)+\left(a_{y}-a_{z}\right)}
$$

And because $C_{f}$ (resp. $C_{g}$ ) is strictly semi-stable (resp. stable) it follows that

$$
\frac{\omega(f, \lambda)}{\left(a_{x}-a_{z}\right)+\left(a_{y}-a_{z}\right)} \leq \frac{d}{3} \quad \text { and } \quad \frac{\omega(h, \lambda)}{\left(a_{x}-a_{z}\right)+\left(a_{y}-a_{z}\right)}<\frac{d}{3}
$$

and hence $\frac{\omega(\mathcal{P}, \lambda)}{\left(a_{x}-a_{z}\right)+\left(a_{y}-a_{z}\right)}<\frac{2 d}{3}$. That is, $\mathcal{P}$ is stable.

Theorem 3.15 If $\mathcal{P} \in \mathscr{P}_{d}$ contains at most two semi-stable curves $C_{f}$ and $C_{g}$ (and all other curves in $\mathcal{P}$ are stable), then $\mathcal{P}$ is strictly semi-stable if and only if there exists a one-parameter subgroup $\lambda$ (and coordinates in $\mathbb{P}^{2}$ ) such that $C_{f}$ and $C_{g}$ are both non-stable with respect to this $\lambda$ that is,

$$
\frac{\omega(f, \lambda)}{\left(a_{x}-a_{z}\right)+\left(a_{y}-a_{z}\right)}=\frac{d}{3} \quad \text { and } \quad \frac{\omega(g, \lambda)}{\left(a_{x}-a_{z}\right)+\left(a_{y}-a_{z}\right)}=\frac{d}{3}
$$

Proof Fix $\mathcal{P}$ as above and note that $\mathcal{P}$ is semi-stable by Corollary 3.12. Next, note that if the two inequalities above hold for some $\lambda$, then $\mathcal{P}$ is strictly semi-stable by Proposition 3.5. Thus, assume $\mathcal{P}$ is strictly semi-stable. Then there exists a one-parameter subgroup $\lambda$ (and 
coordinates in $\left.\mathbb{P}^{2}\right)$ such that $\frac{\omega(\mathcal{P}, \lambda)}{\left(a_{x}-a_{z}\right)+\left(a_{y}-a_{z}\right)}=\frac{2 d}{3}$ and, by Corollary 3.10, it must exist a curve $C_{h}$ in $\mathcal{P}$ such that

$$
\frac{d}{3} \leq \max \left\{\frac{\omega(f, \lambda)}{\left(a_{x}-a_{z}\right)+\left(a_{y}-a_{z}\right)}, \frac{\omega(h, \lambda)}{\left(a_{x}-a_{z}\right)+\left(a_{y}-a_{z}\right)}\right\}
$$

In particular, either $C_{f}$ or $C_{h}$ is non-stable with respect to this $\lambda$. But $C_{f}$ and $C_{g}$ are the only potentially non-stable curves in $\mathcal{P}$. Therefore, either

$$
\frac{\omega(f, \lambda)}{\left(a_{x}-a_{z}\right)+\left(a_{y}-a_{z}\right)} \geq \frac{d}{3}
$$

or

$$
C_{h}=C_{g} \quad \text { and } \quad \frac{\omega(g, \lambda)}{\left(a_{x}-a_{z}\right)+\left(a_{y}-a_{z}\right)} \geq \frac{d}{3}
$$

In any case, we claim that the following equalities hold

$$
\frac{\omega(f, \lambda)}{\left(a_{x}-a_{z}\right)+\left(a_{y}-a_{z}\right)}=\frac{\omega(g, \lambda)}{\left(a_{x}-a_{z}\right)+\left(a_{y}-a_{z}\right)}=\frac{d}{3}
$$

In fact, if $C_{h}=C_{g}$ and (3) holds, then $\frac{\omega(g, \lambda)}{\left(a_{x}-a_{z}\right)+\left(a_{y}-a_{z}\right)}=\frac{d}{3}$ because $C_{g}$ is semi-stable. Thus, by Proposition 3.9, inequality (2) must be true also.

Now, if (2) holds, then $\frac{\omega(f, \lambda)}{\left(a_{x}-a_{z}\right)+\left(a_{y}-a_{z}\right)}=\frac{d}{3}$ because $C_{f}$ is semi-stable. Thus, by Proposition 3.9, we have that $\frac{\omega(h, \lambda)}{\left(a_{x}-a_{z}\right)+\left(a_{y}-a_{z}\right)} \geq \frac{d}{3}$ and, by assumption, it must be the case that $C_{h}=C_{g}$ (and (3) holds).

\section{Stability and the log canonical threshold}

We are now ready to describe how $\omega(\mathcal{P}, \lambda)$ and $\omega(f, \lambda)$ are related to the log canonical threshold of the pair $\left(\mathbb{P}^{2}, C_{f}\right)$. We first recall the following known result and its corollary:

Proposition 4.1 (=[7, Proposition 8.13]) Let $f$ be a holomorphic function near $0 \in \mathbb{C}^{n}$. Assign rational weights $\omega\left(x_{i}\right)$ to the variables $x_{1}, \ldots, x_{n}$ and let $\omega(f)$ be the weighted multiplicity of $f$ (i.e. the lowest weight of the monomials appearing in $f$ ). Then

$$
\operatorname{lct}_{0}\left(\mathbb{C}^{n}, f\right) \leq \frac{\sum \omega\left(x_{i}\right)}{\omega(f)}
$$

Corollary 4.2 Let $C_{f}$ be any plane curve. Then

$$
\frac{\omega(f, \lambda)}{\left(a_{x}-a_{z}\right)+\left(a_{y}-a_{z}\right)} \leq \frac{1}{l c t\left(\mathbb{P}^{2}, C_{f}\right)}
$$

for any one-parameter subgroup $\lambda: \mathbb{C}^{\times} \rightarrow S L(V)$.

Remark 4.3 The main idea behind Corollary 4.2 is that in order to check whether the pair $\left(\mathbb{P}^{2}, t C_{f}\right)$ is $\log$ canonical, it suffices to check that for each weighted blowup of a point $p$ in the plane we have $a\left(E, \mathbb{P}^{2}, t C_{f}\right) \geq-1$, where $E$ denotes the corresponding exceptional divisor (Cf. [4, Section 10]).

Corollary 4.2 together with Corollary 3.10 allow us to conclude that: 
Proposition 4.4 Given a pencil $\mathcal{P} \in \mathscr{P}_{d}$ we have that for any one-parameter subgroup $\lambda: \mathbb{C}^{\times} \rightarrow S L(V)$ there exists $C_{f} \in \mathcal{P}$ such that

$$
\frac{\omega(\mathcal{P}, \lambda)}{\left(a_{x}-a_{z}\right)+\left(a_{y}-a_{z}\right)} \leq \frac{2}{\operatorname{lct}\left(\mathbb{P}^{2}, C_{f}\right)}
$$

And, as a consequence, we recover the statement from Corollary 3.13:

Corollary 4.5 If $\mathcal{P} \in \mathscr{P}_{d}$ is a pencil such that $\operatorname{lct}\left(\mathbb{P}^{2}, C_{f}\right) \geq 3 / d$ (resp. $>$ ) for any curve $C_{f}$ in $\mathcal{P}$, then $\mathcal{P}$ is semi-stable (resp. stable).

Next, we prove the following:

Proposition 4.6 Given $\mathcal{P} \in \mathscr{P}_{d}$ and any base point $p$ of $\mathcal{P}$, there exists a one-parameter subgroup $\lambda: \mathbb{C}^{\times} \rightarrow S L(V)$ (and coordinates in $\mathbb{P}^{2}$ ) such that for any curve $C_{f}$ in $\mathcal{P}$ we have that $\frac{\left(a_{x}-a_{z}\right)+\left(a_{y}-a_{z}\right)}{\omega(\mathcal{P}, \lambda)} \leq l c t_{p}\left(\mathbb{P}^{2}, C_{f}\right)$.

Proof Given $\mathcal{P}$ and a base point $p$, we can always choose coordinates in $\mathbb{P}^{2}$ so that $p=$ $(0: 0: 1)$. We can then consider any one-parameter subgroup $\lambda$, which in these coordinates is normalized as in (1) for some choice of integers $a_{x}, a_{y}$ and $a_{z}$, with $a_{y}-a_{z} \neq 0$. Then $c_{0} \doteq \frac{\left(a_{x}-a_{z}\right)+\left(a_{y}-a_{z}\right)}{\omega(\mathcal{P}, \lambda)} \leq 1$, because $f_{00}=0$ for any curve $C_{f}$ in $\mathcal{P}$ so that we have $m_{00 k l}=0$ for all $0 \leq k, l \leq d$.

We claim that any choice of $\lambda$ as above is such that for any curve $C_{f}$ in $\mathcal{P}$ we have $c_{0} \leq l c t_{p}\left(\mathbb{P}^{2}, C_{f}\right)$.

By contradiction, assume there exists $C_{f}$ in $\mathcal{P}$ such that $\operatorname{lct}_{p}\left(\mathbb{P}^{2}, C_{f}\right)<c_{0}$. Write $\tilde{f}(u, v)=f(x, y, 1)$ and assign weights $\omega(u) \doteq a_{x}-a_{z}$ to the variable $u$ and $\omega(v) \doteq a_{y}-a_{z}$ to the variable $v$ so that the weighted multiplicity of $\tilde{f}$ is precisely $\omega(f, \lambda)$. Now, consider the finite morphism $\varphi: \mathbb{C}^{2} \rightarrow \mathbb{C}^{2}$ given by $(u, v) \mapsto\left(u^{\omega(u)}, v^{\omega(v)}\right)$ and let

$$
\Delta \doteq(1-\omega(u)) H_{u}+(1-\omega(v)) H_{v}+c \cdot \tilde{f}\left(u^{\omega(u)}, v^{\omega(v)}\right)
$$

where $H_{u}$ (resp. $H_{v}$ ) is the divisor of $u=0$ (resp. $v=0$ ) and $c \in \mathbb{Q} \cap[0,1]$.

Then $\varphi^{*}\left(K_{\mathbb{C}^{2}}+c \cdot \tilde{f}(u, v)\right)=K_{\mathbb{C}^{2}}+\Delta$ and by Proposition 5.20 (4) in [8] we know that the pair $\left(\mathbb{C}^{2}, c \cdot \tilde{f}\right)$ is $\log$ canonical at $(0,0)$ if and only if the pair $\left(\mathbb{C}^{2}, \Delta\right)$ is log canonical at $(0,0)$. In particular, taking $c=c_{0}>l c t_{p}\left(\mathbb{P}^{2}, C_{f}\right)=l c t_{0}\left(\mathbb{C}^{2}, \tilde{f}\right)$ it follows that

$$
a\left(E ; \mathbb{C}^{2}, \Delta\right)=-1+\omega(u)+\omega(v)-c \cdot \omega(f, \lambda)<-1
$$

where $E$ is the exceptional divisor of the blow-up of $\mathbb{C}^{2}$ at the origin and $a\left(E ; \mathbb{C}^{2}, \Delta\right)$ is the corresponding discrepancy. But the last inequality is equivalent to the inequality $\omega(\mathcal{P}, \lambda)<$ $\omega(f, \lambda)$, contradicting Proposition 3.5.

Finally, Proposition 4.6 above and Corollary 4.2 together with the other results obtained in this paper, allow us to prove Theorems 4.7 and 4.10 below, thus providing explicit stability criteria.

Theorem 4.7 Let $\mathcal{P}$ be a pencil in $\mathscr{P}_{d}$ which contains a curve $C_{f}$ such that $l$ ct $\left(\mathbb{P}^{2}, C_{f}\right)=\alpha$. If $\mathcal{P}$ is unstable (resp. not stable), then $\mathcal{P}$ contains a curve $C_{g}$ such that lct $\left(\mathbb{P}^{2}, C_{g}\right)<\frac{3 \alpha}{2 d \alpha-3}$ (resp. $\leq$ ). 
Proof If $\mathcal{P}$ is unstable (resp. not stable), then by Proposition 3.2 we can choose a oneparameter subgroup $\lambda$ (and coordinates in $\mathbb{P}^{2}$ ) so that

$$
\frac{2 d}{3}<\frac{\omega(\mathcal{P}, \lambda)}{\left(a_{x}-a_{z}\right)+\left(a_{y}-a_{z}\right)} \quad(\text { resp. } \leq)
$$

By Proposition 3.9, we can find a a curve $C_{g}$ in $\mathcal{P}$ such that

$$
\frac{\omega(\mathcal{P}, \lambda)}{\left(a_{x}-a_{z}\right)+\left(a_{y}-a_{z}\right)} \leq \frac{\omega(f, \lambda)}{\left(a_{x}-a_{z}\right)+\left(a_{y}-a_{z}\right)}+\frac{\omega(g, \lambda)}{\left(a_{x}-a_{z}\right)+\left(a_{y}-a_{z}\right)}
$$

Moreover, by Corollary 4.2 we have that

$$
\frac{\omega(f, \lambda)}{\left(a_{x}-a_{z}\right)+\left(a_{y}-a_{z}\right)} \leq \frac{1}{l c t\left(\mathbb{P}^{2}, C_{f}\right)} \quad \text { and } \quad \frac{\omega(g, \lambda)}{\left(a_{x}-a_{z}\right)+\left(a_{y}-a_{z}\right)} \leq \frac{1}{l c t\left(\mathbb{P}^{2}, C_{g}\right)}
$$

Now, because $\operatorname{lct}\left(\mathbb{P}^{2}, C_{f}\right)=\alpha$, combining the above inequalities we conclude that $\operatorname{lct}\left(\mathbb{P}^{2}, C_{g}\right)<\frac{3 \alpha}{2 d \alpha-3}($ resp. $\leq)$.

Corollary 4.8 Assume $d \geq 5$ and let $\mathcal{P} \in \mathscr{P}_{d}$ be a pencil which contains a smooth member. If any curve $\mathcal{C}_{d}$ in $\mathcal{P}$ is reduced and any point in $\mathcal{C}_{d}$ has multiplicity $m<d$, then $\mathcal{P}$ is stable.

Proof It was proved in [2] that under such conditons any such curve $\mathcal{C}_{d}$ satisfies $l c t\left(\mathbb{P}^{2}, \mathcal{C}_{d}\right) \geq$ $\frac{2 d-3}{(d-1)^{2}}$. Now, because $d \geq 5$ we have that $\frac{2 d-3}{(d-1)^{2}}>\frac{3}{2 d-3}$ and the conclusion follows from Theorem 4.7 with $\alpha=1$.

Corollary 4.9 Let $\mathcal{P} \in \mathscr{P}_{d}$ be a pencil which contains a smooth member. If any curve $\mathcal{C}_{d} \in \mathcal{P}$ contains only points $p$ with multiplicity $m_{p} \leq \frac{2 d-3}{3}$ (resp. $<$ ), then $\mathcal{P}$ is semi-stable (resp. stable).

Proof In fact, any curve $\mathcal{C}_{d}$ in $\mathcal{P}$ satisfies $\frac{1}{m_{p}} \leq \operatorname{lct}\left(\mathbb{P}^{2}, \mathcal{C}_{d}\right)$ (see [7, Lemma 8.10]) and since $\frac{3}{2 d-3} \leq \frac{1}{m_{p}} \quad($ resp. $<)$, the conclusion follows from Theorem 4.7 with $\alpha=1$.

Theorem 4.10 If $\mathcal{P} \in \mathscr{P}_{d}$ is semi-stable (resp. stable), then for any curve $C_{f}$ in $\mathcal{P}$ and any base point $p$ of $\mathcal{P}$ we have $\frac{3}{2 d} \leq l c t_{p}\left(\mathbb{P}^{2}, C_{f}\right)($ resp. $<)$.

Proof Fix $\mathcal{P} \in \mathscr{P}_{d}$ and a base point $p$ as above. Given $C_{f}$ we can always find coordinates in $\mathbb{P}^{2}$ so that $p=(0: 0: 1)$ and we can choose $\lambda$ as in Proposition 4.6. Because $\mathcal{P}$ is semi-stable (resp. stable) for this $\lambda$ we have that

$$
\frac{3}{2 d} \leq \frac{\left(a_{x}-a_{z}\right)+\left(a_{y}-a_{z}\right)}{\omega(\mathcal{P}, \lambda)} \quad(\text { resp. }<)
$$

and the result follows from Proposition 4.6.

Corollary 4.11 Let $\mathcal{P} \in \mathscr{P}_{d}$ be a pencil which contains a curve $\mathcal{C}_{d}$ of the form $m L+C_{d-m}$, where $m L$ is a multiple line with multiplicity $m \geq 2 d / 3$ (resp. $>$ ) and $C_{d-m}$ is a curve of degree $d-m$. Then $\mathcal{P}$ is unstable (resp. not stable). In particular, if $\mathcal{P}$ contains a multiple line with multiplicity $d$, then $\mathcal{P}$ is unstable.

Note that Theorems 4.7 and 4.10 provide explicit stability criteria for a pencil $\mathcal{P} \in \mathscr{P}_{d}$ in terms $\log$ canonical thresholds of pairs $\left(\mathbb{P}^{2}, \mathcal{C}_{f}\right)$, where $C_{f}$ is a curve lying in $\mathcal{P}$. As suggested by the referee, it is interesting to observe that the stability of $\mathcal{P}$ seems to also be related to the $\log$ canonical threshold of the pair $\left(\mathbb{P}^{2}, \mathcal{P}\right)$ - where we extend the notions introduced in Sect. 2 to linear systems as in [7, Definition 4.6]. We prove the following Corollary to Theorem 4.10 and we hope to further investigate this relation in a future project. 
Corollary 4.12 If $\mathcal{P} \in \mathscr{P}_{d}$ is semi-stable (resp. stable), then $\frac{3}{2 d} \leq \operatorname{lct}\left(\mathbb{P}^{2}, \mathcal{P}\right)($ resp. $<)$.

Proof Given $\mathcal{P} \in \mathscr{P}_{d}$, let us denote its general member by $\mathcal{C}^{\text {gen }}$. By [7, Theorem 4.8] we have that $\operatorname{lct}\left(\mathbb{P}^{2}, \mathcal{P}\right)=\operatorname{lct}\left(\mathbb{P}^{2}, \mathcal{C}^{\text {gen }}\right)$. By Bertini's theorem, $\mathcal{C}^{\text {gen }}$ is smooth away from the base points of $\mathcal{P}$, hence $l c t\left(\mathbb{P}^{2}, \mathcal{P}\right)=l c t_{p}\left(\mathbb{P}^{2}, \mathcal{C}^{\text {gen }}\right)$ for some base point $p$. Now, it follows from $\left[7\right.$, Lemma 8.6] that $l c t_{p}\left(\mathbb{P}^{2}, C_{f}\right) \leq l c t_{p}\left(\mathbb{P}^{2}, \mathcal{C}^{\text {gen }}\right)$ for any curve $C_{f}$ in $\mathcal{P}$. As a consequence, Theorem 4.10 implies that whenever $\mathcal{P}$ is semi-stable (resp. stable), then $\frac{3}{2 d} \leq \operatorname{lct}\left(\mathbb{P}^{2}, \mathcal{P}\right)$ (resp. $<$ ).

Acknowledgements I am grateful to my advisor, Antonella Grassi, for her constant guidance, the many conversations and the numerous suggestions on earlier versions of this paper. I am also thankful to the referee for providing insightful comments. This work is part of my $\mathrm{PhD}$ thesis and it was partially supported by a Dissertation Completion Fellowship at the University of Pennsylvania.

Open Access This article is licensed under a Creative Commons Attribution 4.0 International License, which permits use, sharing, adaptation, distribution and reproduction in any medium or format, as long as you give appropriate credit to the original author(s) and the source, provide a link to the Creative Commons licence, and indicate if changes were made. The images or other third party material in this article are included in the article's Creative Commons licence, unless indicated otherwise in a credit line to the material. If material is not included in the article's Creative Commons licence and your intended use is not permitted by statutory regulation or exceeds the permitted use, you will need to obtain permission directly from the copyright holder. To view a copy of this licence, visit http://creativecommons.org/licenses/by/4.0/.

\section{References}

1. Ballico, E., Oliverio, P.: Stability of pencils of plane quartic curves. Atti Accad. Naz. Lincei Rend. Cl. Sci. Fis. Mat. Nat. 74(4), 234-241 (1983)

2. Cheltsov, I.: Worst singularities of plane curves of given degree. J. Geom. Anal. 27(3), 2302-2338 (2017)

3. Dolgachev, I.: Lectures on Invariant Theory: London Mathematical Society Lecture Note Series. Cambridge University Press, Cambridge (2003)

4. Hacking, P.: Compact moduli of plane curves. Duke Math. J. 124(2), 213-257 (2004)

5. Kim, H., Lee, Y.: Log canonical thresholds of semistable plane curves. Math. Proc. Camb. Philos. Soc. 137(2), 273-280 (2004)

6. Kollár, J.: Polynomials with integral coefficients, equivalent to a given polynomial. Electron. Res. Announc. Am. Math. Soc. 3(3), 17-27 (1997). (Electronic only)

7. Kollár, J.: Singularities of pairs. In: Algebraic geometry-Santa Cruz 1995, Proceedings of symposium on Pure Mathematics, vol. 62, pp. 221-287. American Mathematical Society, Providence, RI (1997)

8. Kollár, J., Mori, S.: Birational Geometry of Algebraic Varieties, Cambridge Tracts in Mathematics, vol. 134. Cambridge University Press, Cambridge (2008)

9. Miranda, R.: On the stability of pencils of cubic curves. Am. J. Math. 102(6), 1177-1202 (1980)

Publisher's Note Springer Nature remains neutral with regard to jurisdictional claims in published maps and institutional affiliations. 